\title{
光强对两种入侵植物生物量分配、叶片形态和 相对生长速率的影响
}

\author{
王俊峰1 2 冯玉龙 ${ }^{12 *}$ \\ （1 中国科学院西双版纳热带植物园昆明分部，昆明 650223) (2 河北大学生命科学学院, 保定 071002)
}

\begin{abstract}
摘 要 比较研究了不同光强下生长的(透光率分别为 $12.5 \% 、 36 \% 、 50 \% 、 100 \%$ )两种入侵性不同的外来种一一紫 茎泽兰 (Eupatorium adenophorum) 和兰花菊三七(Gynura sp.)的生物量分配、叶片形态和生长特性。结果表明 : 1) 两 种植物叶片形态对光环境的反应相似。弱光下比叶面积 ( SLA ) 平均单叶面积 $(M L S)$ 和叶面积比 $(L A R)$ 较大, 随着 光强的升高, $S L A 、 M L S 、 L A R$ 和叶根比 ( LARMR) 降低。2) $100 \%$ 光强下紫茎泽兰叶生物量比 ( LMR)、叶重分数 ( $L M F)$ 和叶面积指数高于低光强下的值, 也高于兰花菊三七, 支持结构生物量比 (SBR) 则相反。强光下紫茎泽兰叶片自遮 荫严重, 这可能是其表现入侵性的重要原因之一;兰花菊三七分枝较多, 避免了叶片自遮荫, 较多的分枝利于种子 形成对其入侵有利。3)随生长环境光强的升高, 两种植物的净同化速率 $(N A R)$ 、相对生长速率 $(R G R)$ 和生长对 $N A R$ 的响应系数均升高(但 $100 \%$ 光强下兰花菊三七 $R G R$ 降低), 平均叶面积比 $\left(L A R_{\mathrm{m}}\right)$ 和生长对 $L A R_{\mathrm{m}}$ 的响应系数均降 低, 但不同光强下 $L A R_{\mathrm{m}}$ 对生长的影响始终大于 $N A R$ 。4)随着光强的减弱, 两种植物都增加高度以截获更多光能, 但它们的生物量分配策略不同, 紫茎泽兰根生物量比 $(R M R)$ 降低, $S B R$ 增大, 而兰花菊三七 $S B R$ 降低, RMR 增大。 紫茎泽兰的生物量分配策略更好的反应了弱光环境中的资源变化情况。结论 紫茎泽兰对光环境的适应能力强于 兰花菊三七。
\end{abstract}

关键词 生物量分配 叶片形态 生长特性 紫茎泽兰 兰花菊三七 入侵性

\section{THE EFFECT OF LIGHT INTENSITY ON BIOMASS ALLOCATION , LEAF MORPHOLOGY AND RELATIVE GROWTH RATE OF TWO INVASIVE PLANTS}

\author{
WANG Jun-Feng ${ }^{12}$ and FENG Yu-Long ${ }^{1}{ }^{2}$ * \\ (1 Kunming Division ,Xishuangbanna Tropical Botanical Garden ,Chinese Academy of Sciences ,Kunming 650223 , China) \\ (2 College of Life Science ,Hebei University ,Baoding 071002 , China)
}

\begin{abstract}
The high biodiversity and economic costs associated with invasive plants have promoted research to identify traits associated with invasiveness. At present, most research has been based on comparisons between invasive and native species but, in this paper, two invasive species with different levels of invasiveness, $E u$ patorium adenophorum and Gynura sp. , were studied. Both species were grown under four different relative irradiances $(R I 12.5 \%, 36 \%, 50 \%, 100 \%)$ for 50 days, and we then measured their biomass allocation, leaf morphology and growth properties to compare their light acclimation abilities and growth strategies. The two species exhibited typical leaf morphological responses to different light conditions. At low light levels, plants enhanced light interception by means of increased biomass allocation to leaves and formation of large, thin leaves with high specific leaf area ( $S L A)$, leading to a high leaf area ratio $(L A R)$. At high light levels , plants reduced transpiration losses and increased carbon gain by making small-sized, thick leaves with a low $S L A$, leading to a low $L A R$ and leaf area to root mass ratio. Under most light regimes, $E$. adenophorum was higher in leaf mass ratio $(L M R)$, leaf mass fraction $(L M F), L A R$, root mass ratio $(R M R)$ and root mass to crown mass ratio $(R / C)$, but lower in supporting organ biomass ratio $(S B R), M L A$ and branch number as compared to Gynura sp. Leaf mass ratio , $L M F$, and leaf area index of $E$. adenophorum were the highest under $100 \%$ irradiance than under other light regimes and higher than for Gynura sp. ; however, for SBR, these patterns were reversed. Under $100 \%$ irradiance , leaves were much more self-shaded in $E$. adenophorum than in Gynura sp. This might be an adaptive strategy that supports the vigorous invasiveness of this species because a high-shaded canopy could prevent other plant species from surviving and competing. The number of
\end{abstract}


branches of Gynura sp. was greater under $100 \%$ irradiance than under other light regimes and significantly more than for $E$. adenophorum. This might be an adaptive strategy for Gynura sp. because a greater number of branches can produce more flowers and thus more seeds. For both species, an increase in light intensity resulted in an increase in net assimilation rates $(N A R)$ and growth response coefficient of $N A R\left(G R C_{\mathrm{NAR}}\right)$, whereas mean leaf area ratio $\left(L A R_{\mathrm{m}}\right)$ and growth response coefficient of $L A R_{\mathrm{m}}\left(G R C_{\mathrm{LAR}_{\mathrm{m}}}\right)$ decreased. $G R C_{\mathrm{LAR}_{\mathrm{m}}}$ was higher than $G R C_{\mathrm{NAR}}$ at all times. The relative growth rate $(R G R)$ of $E$. adenophorum increased with an increase in light intensity but decreased in Gynura sp. With a decrease in light intensity, both $E$. adenophorum and Gynura sp. grew taller and produced more branches to intercept more light energy. The biomass allocation strategy differed between the two species: $R M R$ decreased and $S B R$ increased in $E$. adenophorum, while RMR increased, and SBR decreased in Gynura sp. All of the results presented above indicate that $E$. adenophorum was able to acclimate better to different light conditions, especially to low light regimes, than Gynura sp. , and its better ability to acclimate might explain its greater invasiveness .

Key words Biomass allocation, Leaf morphology , Growth characteristic , Eupatorium adenophorum , Gynura sp. , Invasiveness

形态特征和生长特性的改变是植物适应不同环 境和资源水平的重要策略 (Maherali \& Delucia , 2001; Farrar , 1999 ; Müller et al. ,2000)。光是最重要的生 态因子之一(Bazzaz, 1996 ; Poorter , 2001 ; 冯玉龙等， 2002）很多学者对不同光环境下植物的生物量分 配、形态特征、生长速率等作过研究 (King, 1997 ; Poorter, 1999 ; Poorter \& Nagel, 2000 ; 何维明等, 2000 ; 陶建平等, 2003)。生物入侵是生态学研究的 焦点之一(Alpert et al. 2000)。人们发现，入侵植物 一般表现较高的相对生长速率 (RGR)、比叶面积 $(S L A)$ 、叶面积比率 ( LAR ) 和可塑性 (Rejmánek \& Richardson , 1996 ; Williamson \& Fitter , 1996 ; Reichard \& Hamilton , 1997 ; Schweitzer \& Larson , 1999)。这些 结论大都通过比较外来入侵种与土著种得出的, 而 目前普遍认为不同外来种之间的比较对确定与入侵 性有关的特性也很重要 (Alpert et al , ,2000), 但这 种比较尚不多见(Smith \& Knapp，2001)。紫茎泽兰 (Eupatorium adenophorum) 原产中美洲，现已广布于 全球亚热带地区, 是入侵性极强的害草(李振宇等, 2002)。兰花菊三七(Gynura sp.) 是近年传播到西双 版纳地区的，目前表现的入侵性明显弱于紫茎泽兰。 它们都属菊科, 亲缘关系较近。本文以这两种入侵 性不同的外来种为材料, 研究它们的生物量分配、形 态和生长对不同光环境适应策略的异同, 并初步探 讨其与入侵性的关系。

\section{1 材料和方法}

本试验在中国科学院西双版纳热带植物园进 行。该园自然环境概况见文献 (冯玉龙等,2001)。 通过用黑色尼龙网遮荫，建立相对光强( Relative irradiance, $R I)$ 分别为 $4.5 \% 、 12.5 \% 、 36 \% 、 50 \%$ 和
$100 \%$ (不遮荫)的荫棚 5 个。 2002 年 5 月和 7 月分 别将采自西双版纳的紫茎泽兰和兰花菊三七种子播 种在 RI 36\% 的荫棚内的沙床中。同年 8 月, 两种植 物幼苗株高均为 $10 \mathrm{~cm}$ 左右时, 选择大小一致的幼 苗移栽到花盆中, 每盆 1 株, 栽培基质由等体积的林 内 $10 \mathrm{~cm}$ 以上表土(砖红壤)与河沙混匀而成。全部 幼苗在 $36 \%$ 的荫棚中恢复生长 2 周后随机分组, 移 到不同光下再适应 2 周，其中 $12.5 \% 、 36 \% 、 50 \%$ 、 $100 \%$ 光强下各 20 盆, 9月中旬第一次测定生物量， $50 \mathrm{~d}$ 后测定第二次。 $4.5 \%$ 光强下兰花菊三七很快 死亡, 紫茎泽兰虽能够生长但幼苗数量不多, 故两种 植物都没有数据。实验期间每天傍晚浇足水, 每月 施复合肥 1 次, 随时防治病虫害。第一次测定参数 为 株高、总叶面积、叶干重、支持结构干重和根干 重, 第二次比第一次多测分枝数和冠宽。每次测定 选取不同处理下的 2 种植物各 5 株。

用直尺 (精确度 $1 \mathrm{~mm}$ ) 测定株高和冠宽, 其中冠 宽取两个垂直方向测定值的平均数。用 LI-3000 型面 积仪测叶面积。称重部分在 $80{ }^{\circ} \mathrm{C}$ 烘 $48 \mathrm{~h}$, 电子天平 (精确度 $0.0001 \mathrm{~g}$ ) 称量。求出如下参数 根生物量比 (Root mass ratio, RMR ,根重/植株总重); 叶生物量比 (Leaf mass ratio,LMR 叶重/植株总重) ; 支持结构生物 量比(Supporting organs biomass ratio, SBR , 支持结构重 /植株总重) 叶重分数(Leaf mass fraction , $L M F$, 叶重/ 地上部分植株重); 叶面积比 (Leaf area ratio, $L A R$, 总 叶面积/植株总重); 叶根比 (Leaf area root mass ratio , $L A R M R$,总叶面积/总根重);根冠比 (Root mass / Crown mass , $R / C$ 根生物量/地上部分生物量);比叶 面积(Specific leaf area ,SLA 总叶面积/总叶重) ;平均 叶面积 (Mean leaf area ,MLA 总叶面积/叶片数) 冠面 积 (Crown area，0.25 $\pi \times$ 冠宽平方) 叶面积指数 (Leaf 
area index, $L A I$,总叶面积/冠面积); 平均相对生长速 率 $(R G R)$ 净同化速率 $(N A R)$ 、平均叶面积比 $\left(L A R_{\mathrm{m}}\right)$ 和生长响应系数 $(G R C)$ 。 $R G R, N A R, L A R_{\mathrm{m}}, G R C$ 的测定按王沙生等 (1991) 和 Poorter (1999) 的方法。 $R G R=\frac{\ln W_{2}-\ln \bar{W}_{1}}{\Delta_{t}}, N A R=\frac{W_{2}-\bar{W}_{1}}{L_{2}-L_{1}} \times \frac{\ln L_{2}-\ln \bar{L}_{1}}{\Delta_{t}} 。$ 因为 $R G R=L A R_{\mathrm{m}} \times N A R$,所以 $L A R_{\mathrm{m}}=\frac{\ln W_{2}-\ln \bar{W}_{1}}{W_{2}-W_{1}}$ $\times \frac{L_{2}-\bar{L}_{1}}{\ln L_{2}-\ln L_{1}}$ 。其中 $\bar{W}_{1}, \bar{L}_{1}$ 分别表示第一次测定时 某种植物总生物量 $(\mathrm{g})$ 和叶面积 $\left(\mathrm{cm}^{2}\right)$ 的 5 个重复的 算术平均值， $W_{2}, L_{2}$ 分别表示第二次测定时某种植 物单个重复的总生物量 $(\mathrm{g})$ 和叶面积 $\left(\mathrm{cm}^{2}\right) 。 \Delta \mathrm{t}$ 表 示两次测定的时间间隔 $(d)$ 。

用 Sigma Plot 8.0 绘图并拟合趋势线。实验期 间, 两种植物均处于营养生长阶段, 结果有可比性。

\section{2 实验结果}

\section{1 生长光强对两种植物总生物量和株高的影响}

$R I$ 在 $12.5 \% \sim 50 \%$ 范围内, 紫茎泽兰和兰花菊 三七总生物量随光强升高而增加，但光强再高总生物 量反而下降(图 1a) 这与 Poorter(1999)对一些热带树 种的研究结果相似。两种植物的株高均随着光强的 升高而降低(图 1b)。各光下紫茎泽兰总生物量 (12.5\%光下除外)和株高均明显低于兰花菊三七。
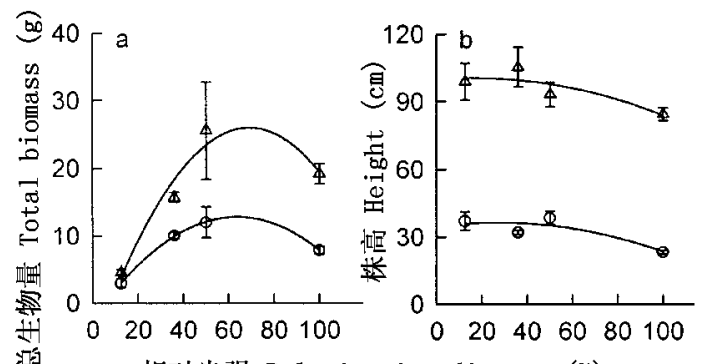

相对光强 Relative irradiances (\%)

○紫茎泽兰 Eupatorium adenophorum $\Delta$ 兰花菊三七 Gynura sp.

图 1 不同光强下生长的紫茎泽兰和兰花菊三七的总生物量和株高 Fig. 1 The total plant biomass and height of Eupatorim adenophorum and Gynura sp. grown in different irradiances

图中数据为 5 次测定的平均值 \pm 标准误 The data were mean $\pm S E$ of 5 separate experiments (图 $2 \sim 5$ 同此 The Fig. 2 to Fig. 5 are the same as Fig. 1)

\section{2 生长光强对两种植物生物量分配相关变量的 影响}

在低光强范围内 $(R I 12.5 \% \sim 36 \%)$, 随着光强 增大 紫茎泽兰 $R M R$ 和 $R / C$ 迅速增大, 光强再高， 增速减缓(图 2a，e)。 $R I$ 在 $12.5 \% \sim 50 \%$ 范围内, 紫 茎泽兰 $S B R$ 和 $L M F$ 变化不大, 光强再高, $S B R$ 迅速 降低, $L M F$ 迅速升高(图 $2 \mathrm{~b}, \mathrm{~d}$ )。紫茎泽兰 $L M R$ 在 中光强下 $(R I 36 \%, 50 \%)$ 较低, 强、弱光下 $L M R$ 较 高(图 2c)。随着光强增大, 兰花菊三七 $R M R 、 R / C$ 、
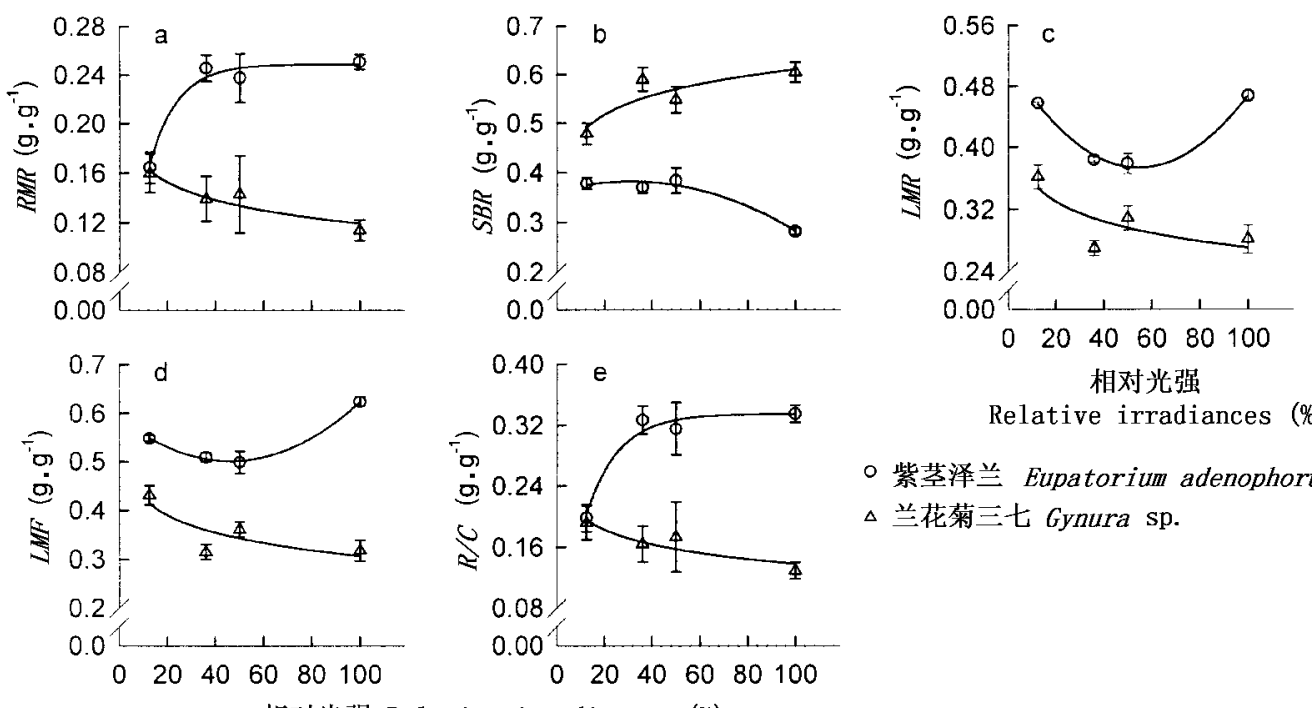

Relative irradiances (\%)

○紫茎泽兰 Eupatorium adenophorum $\Delta$ 兰花菊三七 Gynura sp. 
$L M R$ 和 $L M F$ 减小, $S B R$ 增大 (图 2a e)。除 $12.5 \%$ 光强下 $R M R$ 和 $R / C$ 外, 各光强下兰花菊三七 $S B R$ 高于紫茎泽兰, $R M R 、 L M R 、 L M F$ 和 $R / C$ 则低于紫 茎泽兰。

2.3 生长光强对两种植物 $S L A 、 L A R 、 L A R M R$ 和 $M L A$ 的影响

随着光强的升高两种植物的 $S L A 、 L A R 、 L A R M R$ 和 $M L A$ 均降低 (图 3a d), 这与某些学者的结果相 似(Poorter，1999)。各个光强下紫茎泽兰 $M L A$ 的绝 对值和变幅较兰花菊三七小(图 3d), LAR 高于兰花 菊三七(图 3b)。

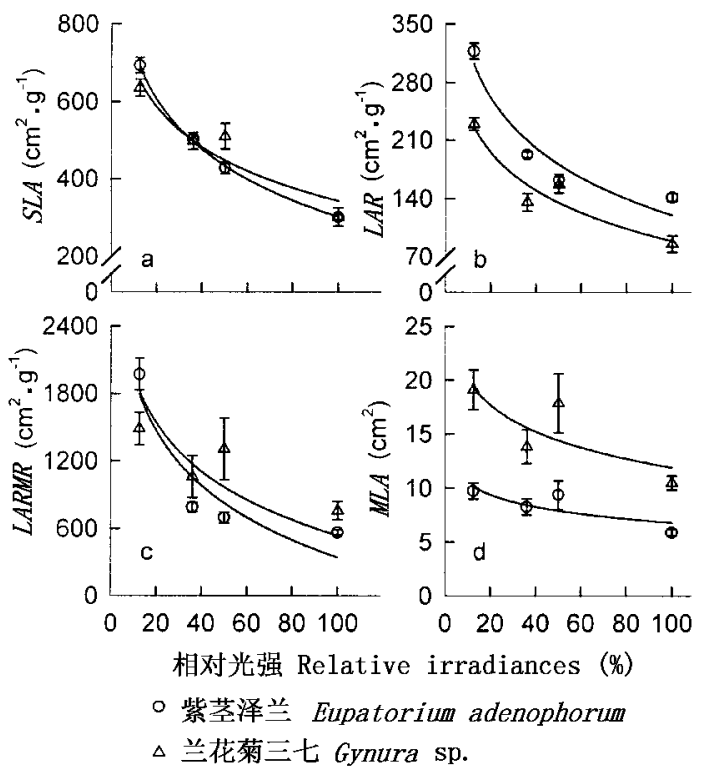

图 3 不同光强下生长的紫茎泽兰和兰花菊三七的比叶面积 $(S L A)$ 、 叶面积比 ( $L A R$ )、叶根比 ( $L A R M R$ ) 和平均单叶面积 $(M L A)$

Fig.3 Specific leaf area $(S L A)$, leaf area ratio $(L A R)$, leaf area root mass ratio ( LARMR) and mean leaf area (MLA) of Eupatorium adenophorum and Gynura sp. grown in different irradiances

2.4 生长光强对两种植物分枝数和叶面积指数的 影响

在低光强范围内 $(R I 12.5 \% \sim 36 \%)$, 紫茎泽兰 和兰花菊三七分枝数随光强升高大幅增加, 光强再 高则增加不明显 (图 4a)。随着光强升高紫茎泽兰 $L A I$ 上升 兰花菊三七 $L A I$ 的变化与光强无明显关 系(图 4b)。100\%光强下兰花菊三七的 $L A I$ 明显小 于紫茎泽兰(图 4b)。

\section{5 生长光强对两种植物生长特性的影响}

随着光强的增加, 两种植物 $N A R$ 均上升, $L A R$ 均下降。紫茎泽兰相对生长速率 $(R G R)$ 在 $R I$ 12.5\% 36\%之间快速增长，RI 36\% 100\%间基本 不变 (图 5a)。兰花菊三七的最大生长速率出现中
等光强, 高光强下 $R G R$ 反而下降 (图 $5 \mathrm{~b}$ )。随着光强 的升高, $N A R$ 对两种植物 $R G R$ 的贡献均增大, $L A R_{\mathrm{m}}$ 的贡献均减小 (图 $5 \mathrm{a} \sim \mathrm{d}$ ), 但各个光强下 $L A R_{\mathrm{m}}$ 始终 是 $R G R$ 变化的主要诱因 (图 $5 \mathrm{c}, \mathrm{d}$ )。
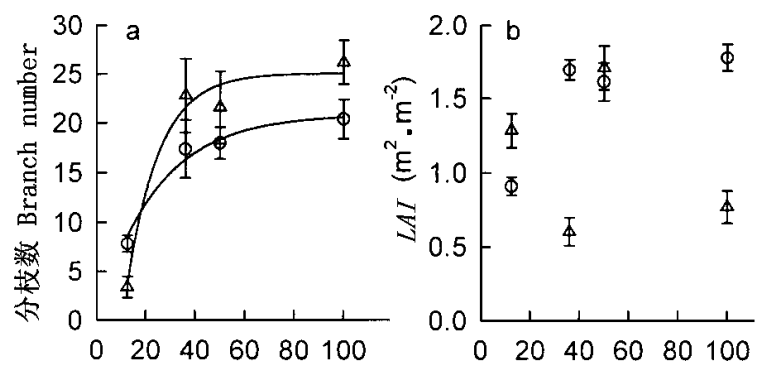

相对光强 Relative irradiances (\%)

○紫茎泽兰 Eupatorium adenophorum

$\Delta$ 兰花菊三七 Gynura sp.

图 4 不同光强下生长的紫茎泽兰和兰花菊 三七的分枝数和叶面积指数 $(L A I)$

Fig.4 The branch number and leaf area index (LAI) of Eupatorium adenophorum and Gynura sp. grown in different irradiances

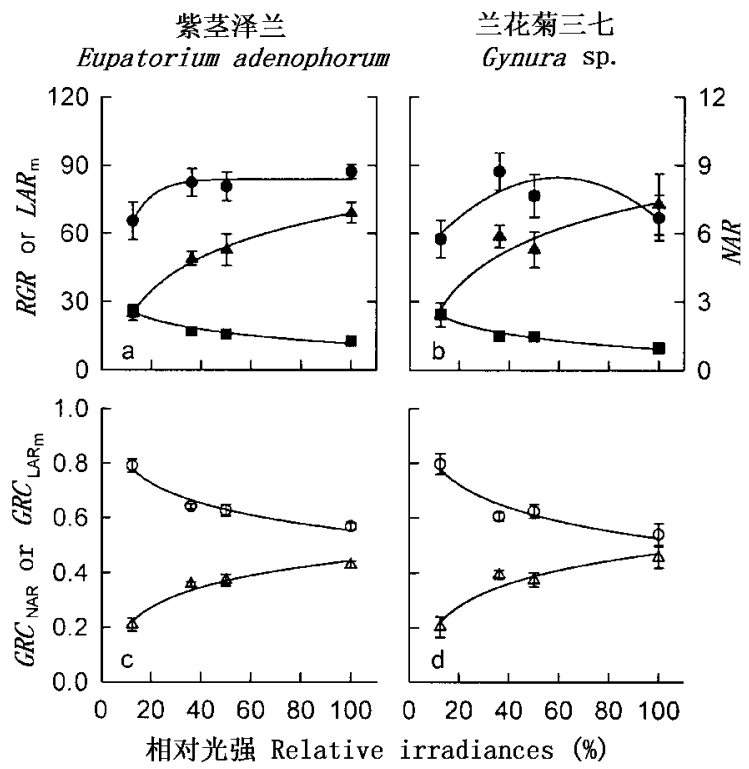

- $R G R$ - $L A R_{\mathrm{m}} \perp N A R \quad \triangle G R C_{\mathrm{NaR}} \circ G R C_{\mathrm{LAR}_{\mathrm{m}}}$

图 5 不同光强下生长的紫茎泽兰和兰花菊三七的 $R G R$ 、 $N A R 、 L A R$ 和 $G R C$

Fig.5 The RGR , NAR , LAR and GRC of Eupatorium adenophorum and Gynura sp. grown in different irradiances

$R G R$ : 平均相对生长速率 Mean relative growth rate $\left(\mathrm{mg} \mathrm{g}^{-1} \cdot \mathrm{d}^{-1}\right)$ $N A R$ : 净同化速率 Net assimilation rate $\left(\mathrm{g} \mathrm{m}^{-2} \mathrm{~d}^{-1}\right) \quad L A R_{\mathrm{m}}$ : 平均叶 面积比 Mean leaf area ratio $\left(\mathrm{m}^{2} \mathrm{~kg}^{-1}\right) \quad G R C_{\mathrm{NAR}}$ : 生长对 $N A R$ 的响应 系数 The growth response coefficients of $N A R$, that means the contribution of $N A R$ to $R G R \quad G R C_{\mathrm{LAR}_{\mathrm{m}}}$ : 生长对 $L A R_{\mathrm{m}}$ 的响应系数 The growth response coefficients of $L A R_{\mathrm{m}}$, that means the contribution of $L A R_{\mathrm{m}}$ to $R G R$ 


\section{3 讨 论}

弱光下紫茎泽兰和兰花菊三七均增加叶片生物 量投入(图 2c ,d) 增大 $S L A$ (图 3a) 和 $L A R$ (图 3b), 提高了捕光能力。随着光强的升高它们的叶片变小 (图 3d)、变厚、SLA 降低 (图 3a)。厚的叶片光合能 力强(Oberbauer \& Strain , 1984 ; Poorter et al ., 1995 ; 冯玉龙等，2002），小叶散热快、蒸腾失水少 (Gibson， 1998)。强光下单位总生物量和单位根生物量所承 载的叶面积明显减少(图 $3 \mathrm{~b}, \mathrm{c}$ ), 这有利于植株保持 强光下的水分平衡，但两者采取的策略不同。

随着光强的升高植物倾向于提高 $R M R$ 以汲取 更多水分 (Poorter, 1999 ; 何维明等, 2000 ; Poorter \& Nagel，2000）紫茎泽兰就是这样(图 2a)。但强光下 兰花菊三七 $R M R$ 却下降了 (图 2a)。为了维持水分 平衡, 兰花菊三七必须减少蒸腾器官 (主要是叶) 的 生物量比(图 2c) 其结果是 SBR 上升(图 2b), 并形 成了大量分枝 (图 4a)。叶片松散排列在不同的分 枝上避免了叶片间的相互遮荫, 表现为 $L A I$ 很低(图 $4 \mathrm{~b})$ 。避免遮荫可以最大限度的发挥叶片的光合能 力从而提高 $N A R$ (图 5b)。研究表明, 强光下兰花 菊三七叶片光合速率很高而光抑制程度很低 (王俊 峰等 2003)。但强光下高的光合速率和 $N A R$ 并不 足以弥补 $L A R$ 下降对 $R G R$ 造成的影响 (图 $5 \mathrm{~b}$ )。较 多的分枝为产生更多的种子提供了条件，因为每个 枝的顶端都会发育成生殖器官, 形成种子 较高的光 合速率可以保证兰花菊三七用较少的叶片制造出供 种子发育所需的养分。 $R M R$ 降低可能会导致植株 水分胁迫; $L M R$ 降低、 $S B R$ 增加导致同化组织与非 同化组织比例降低, 进而降低植株生长速度和耐荫 能力。那么, 强光下兰花菊三七为什么要冒着水分 胁迫的危险、付出降低 $R G R$ 和耐荫能力的代价减少 根和叶生物量分配，增加枝的生物量分配? 作者认 为这是兰花菊三七的生殖策略, 为产生更多的种子， 它不得不做出某些牺牲。生殖生长和营养生长之间 的权衡 (Trade-off) 是常见的现象 (McDowell \& Turner， 2002 ）,但兰花菊三七这种类型的生殖和营养生长的 权衡尚属首次报道。尽管如此，兰花菊三七的种子 数量还是远远少于紫茎泽兰, 这可能也是其入侵性 较弱的原因之一。

强光下紫茎泽兰的分枝较少 (图 4a), 但其分枝 多发自根茎交汇处，分枝密集且高度相近均明显低 于兰花菊三七(图 1b), 叶片较多, 但面积较小(图 $3 \mathrm{~d}$ ), $L A I$ 较高(图 4b) , 导致相当部分叶面积位于冠
内 这些因素都会使其叶片自遮荫较兰花菊三七严 重得多。作者曾经发现 $100 \%$ 光强下紫茎泽兰会发 生较明显的日间光抑制,但其耐荫能力较强,在 $4.5 \%$ 光强下仍有较强的光合能力 (王俊峰等, 2004)。我们认为自遮荫是紫茎泽兰适应强光的有 效策略, 自遮荫可以缓解叶片的光抑制, 强光下牺牲 叶片的部分光合能力可以避免整株水平上碳积累的 减少。野外考察发现, 紫茎泽兰往往为成片密集的 单优群落,其冠层下透光率约为 $1.8 \%$ (未发表数 据) , 其它植物很难进入。据此推测自遮荫可能对其 入侵性的表现至关重要。高度自遮荫可以阻止其它 植物在其冠内生长, 也可以逐步排挤其定植地周围 的其它植物。Yamashita 等(2002)认为生理可塑性较 高适度耐荫的植物成为入侵种的可能性最大。外来 物种可以通过遮荫排挤本地植物种 (Standish et al. , 2001 ; Niinemets et al .,2003)。紫茎泽兰具有这样 的特性。

随着光强的减弱, 两种植物均通过减少分枝(图 4a)、增加株高 (图 1b)向高空发展以截获更多光能。 紫茎泽兰削减根的生物量来补给支持结构 (图 2a c)，这种分配方式可以降低 $L A I($ 图 4b) 减少自遮荫， 还可以降低 $R / C$ (图 2e)，这与光能减少和蒸腾减弱 相适应。而兰花菊三七则将生物量从支持结构分配 到了叶和根中 (图 $2 \mathrm{a} \sim \mathrm{c}$ ), $L M R$ 的增大 (图 2c) 有利 于捕光, 低光下 $R M R$ 的增加 (图 2a) 似乎与根的吸 收和支持功能关系不大 其意义还需进一步研究。

综上, 两种植物都能适应变幅较大 $(R I 12.5 \%$ $\sim 100 \%$ ) 的光环境, 且都能很好地适应强光环境。 而弱光下, 紫茎泽兰生物量的分配策略比兰花菊三 七更能反应环境的改变。我们还发现 $4.5 \%$ 光强下 紫茎泽兰可以生长, 而兰花菊三七很快死亡。可见， 总体上讲紫茎泽兰对光环境的适应能力强于兰花菊 三七, 这可能是目前紫茎泽兰入侵性强于兰花菊三 七的原因之一。

\section{参 考 文 献}

Alpert, P., E. Bone \& C. Holzapfel. 2000. Invasiveness, invisibility and the role of environmental stress in the spread of non-native plants. Perspectives in Plant Ecology, Evolution and Systematics, 3: $52 \sim 66$.

Bazzaz, F. A. 1996. Plant in changing environments: linking physiological, population and community ecology. London: Cambridge University Press. $13 \sim 40$.

Farrar, J. F. 1999. Acquisition, partitioning and loss of carbon. In: Press, M.C., J. D. Scholes \& M.G. Barker eds. Physiological plant ecology. Oxford: Blackwell. 25 43.

Feng，Y. L. (冯玉龙)，Z． L. Feng(冯志立) \& K. F. Cao(曹 
坤芳). 2001. The protection against photodamage in Amomum villosum Lour. Acta Phytophysiologica Sinica(植物生理学报), 27: $483 \sim 488$. (in Chinese with English abstract)

Feng, Y. L. (冯玉龙), K. F. Cao(曹坤芳), Z. L. Feng(冯志 立) \& L. Ma (马玲) . 2002. Acclimation of lamina mass per unit area, photosynthetic characteristics and dark respiration to growth light regimes in four tropical rainforest species. Acta Ecologica Sinica (生态学报), 22: $901 \sim 910$. (in Chinese with English abstract)

Gibson, A. C. 1998. Photosynthetic organs of desert plants. BioScience, 48: $911 \sim 920$.

He, W. M. (何维明) \& Z. C. Zhong(钟章成). 2000. Morphological and growth responses of the climbing plant Gynostemma pentaphyllum seedlings to varying light intensity. Acta Phytoecologica Sinica(植物生态学报), 24: $375 \sim 378$. (in Chinese with English abstract)

King, D. A. 1997. Branch growth and biomass allocation in Abies amabilis saplings in contrasting light environments. Tree Physiology, 17: $251 \sim 258$.

Li, Z. Y. (李振宇) \& Y. Xie (解炎). 2002. Invasive alien species in China. Beijing: China Forestry Publishing House. 163. (in Chinese)

Maherali, H. \& E. H. DeLucia. 2001. Influence of climate-driven shifts in biomass allocation on water transport and storage in ponderosa pine. Oecologia, 129: $481 \sim 489$.

McDowell, S. C. L. \& D. P. Turner. 2002. Reproductive effort in invasive and non-invasion Rubus. Oecologia, 133: $102 \sim 111$.

Müller, I., B. Schmid \& J. Weiner. 2000. The effect of nutrient availability on biomass allocation patterns in 27 species of herbaceous plants. Perspectives in Plant Ecology, Evolution and Systematics, 3: $115 \sim 117$.

Niinemets, Ü., F. Valladares \& R. Ceulemans. 2003. Leaf-level phenotypic variability and plasticity of invasive Rhododendron ponticum and non-invasive Ilex aquifolium co-occurring at two contrasting European sites. Plant, Cell and Environment, 26: $941 \sim 956$

Oberbauer, S. F. \& B. R. Strain. 1984. Photosynthesis and successional status of Costa Rica rain forest trees. Photosynthesis Research, 5: $227 \sim 232$.

Poorter, L. 1999. Growth responese of 15 rain-forest tree species to a light gradient: the relative importance of morphological and physiological traits. Functional Ecology, 13: $396 \sim 410$.

Poorter, L. 2001. Light-dependent changes in biomass allocation and their importance for growth of rain forest tree species. Functional Ecology, 15: $113 \sim 123$.

Poorter, H., S. F. Oberbauer \& D. B. Clark. 1995. Leaf optical properties along a vertical gradient in a tropical rain forest canopy in Costa Rica. American Journal of Botany, 82: $1257 \sim 1263$.

Poorter, H. \& O. Nagel. 2000. The role of biomass allocation in the growth response of plant to different levels of light, $\mathrm{CO}_{2}$, nutrients and water: a quantitative review. Australian Journal of Plant Physiology, 27: 595 607.

Reichard, S. H. \& C. W. Hamilton. 1997. Predicting invasions of woody plant introduced into North America. Conservation Biology, 11: $193 \sim 203$.

Rejmánek, M. \& D. M. Richardson. 1996. What attributes make some plant species more invasive? Ecology, 77: 1655 1661 .

Schweitzer, J. A. \& K. C. Larson. 1999. Great morphological plasticity of exotic honeysuckle species may make them better invaders than native species. Journal of the Torrey Botanical Society, 126: $15 \sim 23$.

Smith, M. D. \& A. K. Knapp. 2001. Physiological and morphological traits of exotic, invasive exotic, and native plant species in tallgrass prairie. International Journal of Plant Sciences, 162: $785 \sim 792$.

Standish, R. J., A. W. Robertson \& P. A. Williams. 2001. The impact of an invasive weed Tradescantia fluminensis on native forest regeneration. Journal of Applied Ecology, 38: 1253 1263 .

Tao, J. P. (陶建平) \& Z. C. Zhong (钟章成). 2003. Effect of light on morphological plasticity and biomass allocation of Momordica charantia. Chinese Journal of Applied Ecology (应用生 态学报), 14: 336 340. (in Chinese with English abstract)

Wang, J. F. (王俊峰), Y. L. Feng (冯玉龙) \& Z. Li (李志). 2003. Acclimation of photosynthesis to growth light intensity in Chromolaena odorata (L.) and Gynura sp. Journal of Plant Physiology and Molecular Biology (植物生理与分子生物学学 报)，29: 542 548. (in Chinese with English abstract)

Wang, J. F. (王俊峰) , Y. L. Feng (冯玉龙) \& H. Z. Liang(梁 红柱). 2004. Acclimation of photosynthetic characteristics to growth light intensity in Eupatorium adenophorum Spreng. Chinese Journal of Applied Ecology(应用生态学报), 15: 1373 1377. (in Chinese with English abstract)

Wang, S. S. (王沙生) , R. F. Gao(高荣孚) \& G. M. Wu(吴 贯明). 1991. Plant physiology. Beijing: China Forestry Publishing House. $284 \sim 286$. (in Chinese)

Williamson, M. \& A. Fitter. 1996. The characters of successful invaders. Biological Conservation, 78: $163 \sim 170$.

Yamashita, N., N. Koike \& A. Ishida. 2002. Leaf ontogenetic dependence of light acclimation in invasive and native subtropical trees of different successional status. Plant, Cell and Environment, 25: $1341 \sim 1356$. 